\title{
Caractérisation des pratiques locales du mil Sanio [Pennisetum glaucum (L.) R. Br] en zone soudanienne humide au Sénégal
}

\author{
Baboucar BAMBA ${ }^{1 *}$, Moustapha GUEYE ${ }^{2}$, Daouda $\mathrm{NGOM}^{3}$, Samba Laha KA ${ }^{4}$, \\ Bathé DIOP ${ }^{1}$ et Ghislain KANFANY ${ }^{2}$
}

\author{
${ }^{1}$ Institut Sénégalais de Recherches Agricoles Centre de Recherches Agricoles de Djibélor, BP 34. \\ ${ }^{2}$ Institut Sénégalais de Recherches Agricoles, Centre National de Recherches Agronomiques de Bambey, \\ BP 211. \\ ${ }^{3}$ Laboratoire d'agro-écologie, Département de Biologie Végétale Faculté des Sciences et Techniques, \\ Université Cheikh Anta DIOP, B.P.5005 Dakar-Fann, Sénégal. \\ ${ }^{4}$ Laboratoire de Botanique et Biodiversité, Département de Biologie Végétale, Faculté des Sciences et \\ Techniques, Université Cheikh Anta DIOP, B.P.5005 Dakar-Fann, Sénégal. \\ *Auteur correspondant ; E-mail: beubeuz04@yhaoo.fr, Tél: +2215854262.
}

\section{RESUME}

Le mil sanio ou type tardif [Pennisetum glaucum (L.) R.Br)] est une céréale négligée et cultivée essentiellement dans les régions sud et sud-est du Sénégal. Il est très apprécié par les populations rurales à cause de ses qualités organoleptiques et fourragères. Une étude des pratiques culturales du mil sanio a été effectuée dans 26 parcelles paysannes réparties dans 11 villages et 5 communes (Ndorna, Bignarabe, Medina El hadj, Guiro yero Bocar, et Dioulacolon) de la région de Kolda en Haute Casamance. Les résultats ont montré que le mil sanio est plus cultivé dans les champs de case (65\%) que dans les autres champs. Les superficies emblavées dépassent rarement 2 ha $(84 \%)$. Le semis à plat est largement pratiqué $(92 \%)$ et la période de semis est calée pendant la deuxième moitié de juin $(43 \%)$ et de juillet $(34 \%)$. La pratique de l'amendement (38\%) et de la fertilisation (42\%) n'est pas courante dans la zone. Les principales adventices rencontrées dans les champs de mil sanio sont par ordre d'importance Digitaria horizontalis Willd, Pennisetum pedicellatum Trin. et le striga hermontica (Delile.) Benth. Le désherbage manuel et mécanique est majoritairement effectué $(72 \%)$. Les rendements en grains les plus élevés sont enregistrés dans la partie la plus arrosée de la zone d'étude (communes de Médina El Hadji $626 \pm 133 \mathrm{~kg} \mathrm{ha}^{-1}$; Guiro Yero Bocar $630 \pm 167$ $\mathrm{kg} \mathrm{ha}^{-1}$ et Bignarabe $540 \pm 63 \mathrm{~kg} \mathrm{ha}^{-1}$ et Dioulacolon $\left.517 \pm 244 \mathrm{~kg} \mathrm{ha}^{-1}\right)$. Par contre les plus faibles rendements sont notés dans la zone la moins pluvieuse de la zone d'étude (commune de Ndorna $369 \pm 104 \mathrm{~kg} \mathrm{ha}^{-1}$ ). Ces premiers résultats contribueront à l'établissement d'un référentiel technique sur la culture du mil sanio. Des expérimentations sur la période de semis, les écartements de semis, la fertilisation organo-minérale sont fortement recommandées afin d'intensifier les itinéraires techniques et améliorer les rendements du mil sanio. (C) 2019 International Formulae Group. All rights reserved

Mots clés: Mil sanio, Pennisetum glaucum, pratiques locales, Haute Casamance, Sénégal

\section{Characterization of local practices of Sanio millet [Pennisetum glaucum (L.) R. $\mathrm{Br}$ ] in wet Sudanese zone in Senegal}

\section{ABSTRACT}

Sanio millet [Pennisetum glaucum (L.) R. Br] is a neglected cultivated cereal mainly grown in the southern and south-eastern regions of Senegal. It is very appraised by smallholders farmers because of his grain 
and fodder qualities. A field survey conducted on pearl millet cultural practices was conducted in 26 farmer's plots selected in 11 villages. These villages are located in 5 communities (Ndorna, Medina El hadj, Guiro yero Bocar, Bignarabe and Dioulacolon) in Kolda Region. Results showed that sanio millet is more cultivated in fields located near the houses $(65 \%)$. Cultivated pearl millet areas are most of the time less than 2 ha $(84 \%)$. The flat sowing is broadly practiced (92\%) and is mainly done between June (43\%) and July (34\%). Application of manure (38\%) and mineral fertilizer (42\%) in not common in this part of the country. The main weeds encountered in millet sanio fields are in order of importance Digitaria horizontalis Willd, Pennisetum pédicellatum Trine. and the Striga hermontica (Delile.) Benth. The manual and mechanical weeding is mainly done $(72 \%)$. Highest grain yield were recorded in Médina El Hadji $\left(626 \pm 133 \mathrm{~kg} \mathrm{ha}^{-1}\right)$; Guiro Yero Bocar (630 $\left.\pm 167 \mathrm{~kg} \mathrm{ha}^{-1}\right)$ and Bignarabe $\left(540 \pm 63 \mathrm{~kg} \mathrm{ha}^{-1}\right)$ and Dioulacolon $\left(517 \pm 244 \mathrm{~kg} \mathrm{ha}^{-1}\right)$, located in wet zone. While low yields were recorded in the driest area at Ndorna $\left(369 \pm 104 \mathrm{~kg} \mathrm{ha}^{-1}\right)$. These preliminary results will contribute to the establishment of a technical reference for late pearl millet called Sanio. Experimentations on the period of sowing, row spacing, organo-mineral fertilization are strongly recommended in order to contribute to the yield improvement of late millet.

(C) 2019 International Formulae Group. All rights reserved

Keyswords: Sanio millet, Pennisetum glaucum cultural practices, High Casamance, Sénégal

\section{INTRODUCTION}

$\mathrm{Au}$ Sénégal Oriental et en Haute Casamance, les céréales occupent $55 \%$ des superficies emblavées dont $50 \%$ en mil et sorgho (Fofana et al., 2009). Le mil occupe une place très importante dans l'alimentation humaine en zones rurale et urbaine En 2013, la production de mil dans la région de Kolda était estimée à 13908 tonnes sur une superficie de 16683 ha avec un rendement moyen de $834 \mathrm{~kg} / \mathrm{ha}$ (ANSD, 2014).

Deux types de mil sont cultivés au Sénégal: le mil souna ou type hâtif est presque cultivé sur tout le territoire national (ISRA et al., 2005), par contre, le type tardif ou mil sanio est essentiellement cultivé dans les régions sud et sud-est du pays. La faiblesse des rendements en grain du mil $(750 \mathrm{~kg} / \mathrm{ha})$ est essentiellement due à la combinaison de facteurs abiotique (déficit pluviométrique, pauvreté des sols, matériel local rustique et peu productif), biotique (insectes ravageurs, maladies, adventices) et à l'inadéquation des pratiques culturales (Kouakou et al., 2013). Les années de sécheresse 1970-1973 ont orienté les recherches en amélioration variétale vers la création des variétés hâtives telles que le Souna 3 vulgarisé dans la zone centre-sud et est (Fall et Lô, 2009). Des travaux de recherches sur les variétés précoces ont concerné le système de culture et la protection des cultures (Bagayoko et al.,
2000 ; ROCAFREMI, 2002 ; ISRA et al., 2005). Contrairement au type souna, le mil sanio n'a pas été suffisamment accompagné par la recherche (inexistence de variétés améliorées, absence de référentiel technique et une méconnaissance des pratiques locales). Le mil sanio qui est apprécié par les populations rurales à cause de ses qualités organoleptiques et fourragères pourrait s'intégrer dans un système de diversification des cultures. L'amélioration des techniques de culture de ce type de mil en Casamance et au Sénégal Oriental pourrait accroitre les rendements dans un contexte de variabilité climatique. Cette présente étude a pour objectif spécifique d'analyser les pratiques culturales du mil sanio en Haute Casamance.

\section{MATERIEL ET METHODE Zone d'étude}

L'étude a été conduite en Haute Casamance, sous-zone agro-écologique située au Sud du Sénégal Figure 1. Le climat est de type sud soudanien continental (Sagna et al., 2012). Le cumul pluviométrique était de 1070 mm en 2015 et sur la période 1981-2010, la normale climatique était de $1191 \mathrm{~mm}$. Les sols sont de types ferrugineux tropicaux lessivés au niveau des plateaux (ANSD, 2015). 


\section{Echantillonnage}

Le choix des villages et des producteurs a été effectué en fonction de l'importance de la production du mil et de l'accessibilité des parcelles des producteurs en période d'hivernage Tableau 1. Le dispositif de suivi parcellaire a été installé dans 26 parcelles paysannes réparties dans 11 villages et cinq (5) communes de la région de Kolda. Dans chaque parcelle, 5 carrés de $10 \mathrm{~m}$ de coté au niveau des 4 angles et au centre de la parcelle ont été délimités Figure 2.

\section{Observations et mesures}

Les paramètres suivis ont été collectés à l'aide d'un questionnaire d'enquête et d'une fiche de suivi agro morphologique et de production. Le questionnaire a mis l'accent sur les pratiques culturales des producteurs (superficie des parcelles, période et mode de semis, désherbage, type de et dose de fertilisation). Le suivi parcellaire a permis de collecter les données relatives au tallage, à la diversité floristique, au rendement et ses composantes.
Les espèces d'adventices ont été identifiées grâce à l'utilisation de guides sur les adventices tropicales (Berhaut, 1967 ; Merlier et Montegut, 1982). La nomenclature employée est celle de Lebrun et Stork (1997). Pour les types biologiques, nous avons utilisé la classification de Raunkier (1934) adaptée à la zone tropicale où la saison défavorable correspond à la saison sèche (Lebrun, 1966 ; Trochain, 1966). Cette classification distingue 6 formes biologiques qui sont : les nanophanérophytes $(\mathrm{P})$, les chaméphytes $(\mathrm{C})$, les hémicryptophytes $(H)$, les géophytes $(\mathrm{G})$, les thérophytes $(\mathrm{T})$ et les plantes parasites (Par).

\section{Analyse statistique}

Les données collectées ont été saisies à l'aide du tableur EXCEL. Les analyses ont porté sur les statistiques descriptives (moyenne, écart type, fréquence). Ces analyses ont été effectuées avec le logiciel Statistical Package for Social Sciences (SPSS) version 17.

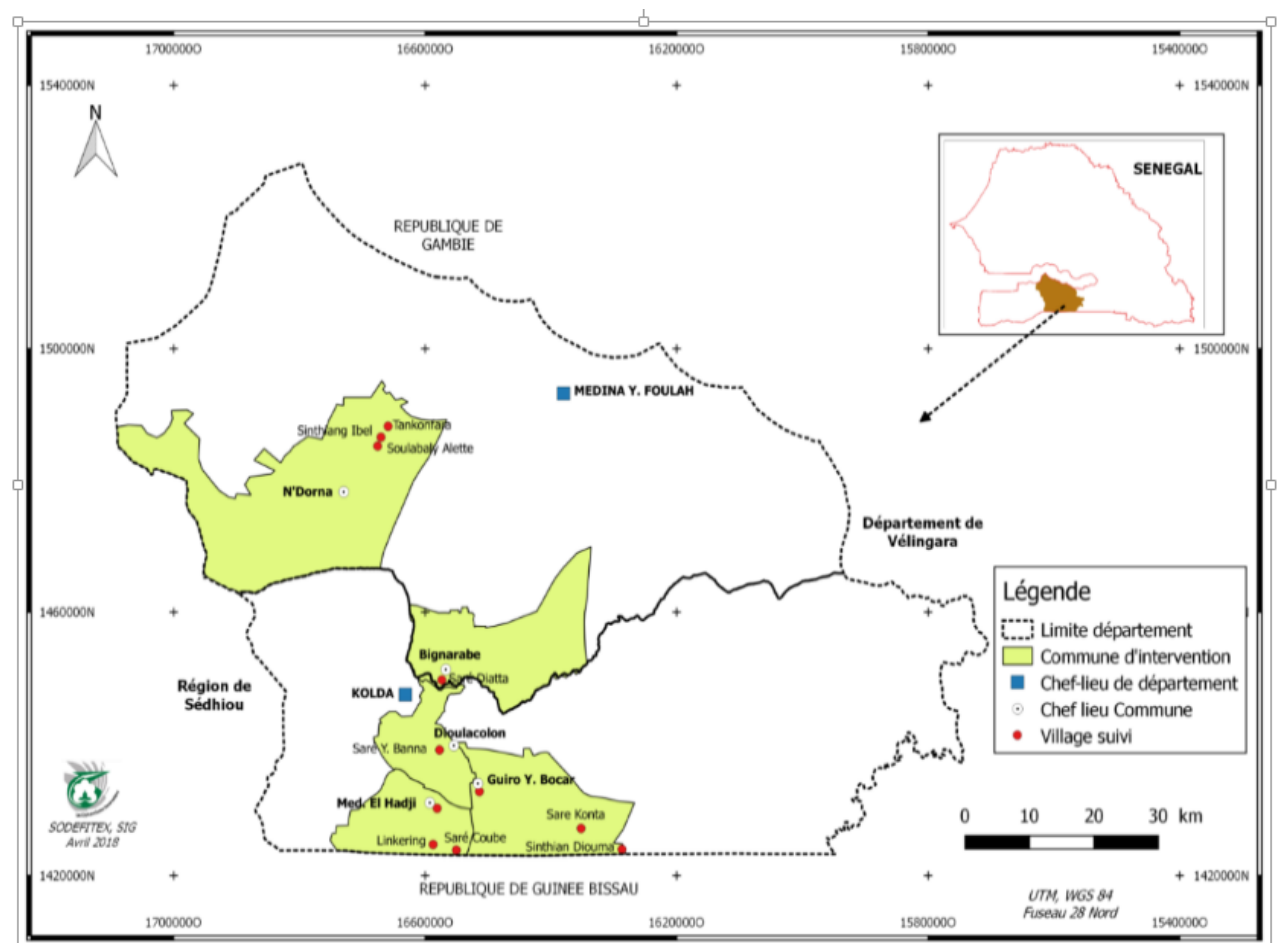

Figure 1: Localisation des sites du suivi des pratiques culturales. Source : SODEFITEX. 
Tableau 1 : Répartition des producteurs par village et par commune.

\begin{tabular}{llll}
\hline Départements & Communes & Villages & Nombre de producteurs \\
\hline \multirow{4}{*}{ Kolda } & \multirow{2}{*}{ Medina El hadj } & Medina El Hadj & 2 \\
& & Saré Coubé & 1 \\
& & Linkering/Kamako & 3 \\
\cline { 2 - 4 } & Dioulacolon & Saré Yero Bana & 5 \\
\cline { 2 - 4 } & \multirow{3}{*}{ Guiro Yero Bocar } & Guiro Yero Bocar & 2 \\
& & Sinthian Diouma & 2 \\
& & Sare Conta & 1 \\
\hline \multirow{3}{*}{ Médina Yoro Foula } & Bignarabe & Saré Diatta & 5 \\
\cline { 2 - 4 } & \multirow{2}{*}{ Ndorna } & Tancofara & 3 \\
& & Sourabaly Alette & 1 \\
\hline Total & & Sinthian Ibel & 1 \\
\hline
\end{tabular}

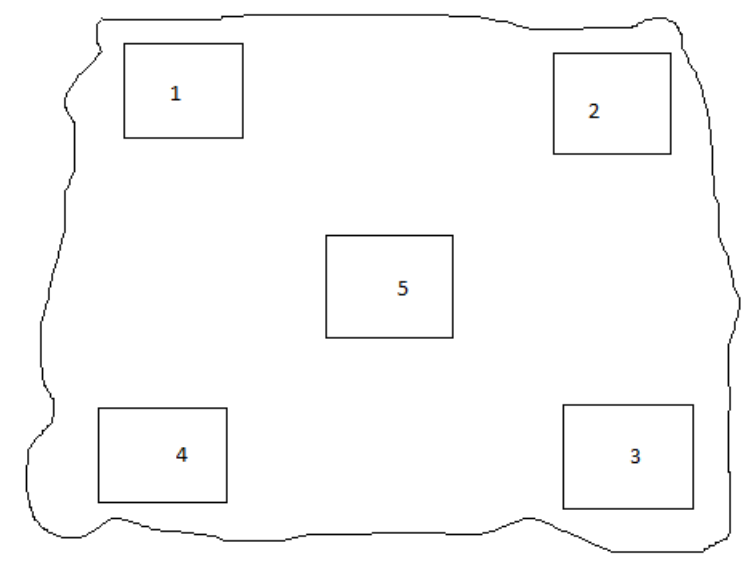

Figure 2: Schéma simplifié du dispositif de suivi des parcelles.

\section{RESULTATS}

\section{Caractéristiques des champs de mil sanio}

Le Tableau 2 montre la répartition des parcelles de mil sanio en fonction du type de champ et de superficie moyenne emblavée. Le mil sanio est cultivé dans les champs de case proches des concessions et dans les champs de brousse qui sont éloignés du village. Soixantesept $(67 \%)$ des parcelles sont en brousse. Les superficies par exploitation varient en moyenne entre 1 et 3 ha. Plus de $80 \%$ des parcelles ont une superficie inférieure à 2 ha.

L'arachide est le principal précédent cultural (50\%), suivi du maïs (23\%). La monoculture et la jachère sont par contre des pratiques rares dans la zone d'étude.
Cependant, sur $60 \%$ des parcelles à Bignarabe, la culture continue du mil sanio a été observée Tableau 3.

\section{Période et mode de semis}

Le semis à plat est majoritairement pratiqué dans les parcelles (93\%) alors que le semis sur billons n'est observé que dans la localité de Médina El Hadji (33\%). Le semis du mil sanio est généralement effectué dans la deuxième quinzaine des mois de juin et de juillet avec $43 \%$ et $34 \%$ des parcelles suivies Tableau 4.

\section{Amendement et fertilisation}

Les pratiques de fertilisation et d'amendement sont consignées dans le Tableau 5. Ainsi $23 \%$ et $19 \%$ des producteurs 
ont utilisé respectivement de l'engrais NPK et de l'urée tandis que $38 \%$ ont fumé leurs parcelles avec de l'engrais organique. En général, les producteurs épandent l'engrais minéral (NPK 15-15-15 et Urée $46 \% \mathrm{~N}$ ) avant épiaison et procèdent à un recouvrement immédiat (Tableau 6).

\section{Inventaire et gestion des nuisibles}

Le Tableau 7 indique la fréquence d'occurrence des différentes espèces d'adventices identifiées dans les parcelles de mil sanio. Ainsi les espèces Digitaria horizontalis Willd, $(88,5 \%)$ Pennisetum pedicellatum Trin $(57,7 \%)$ et Striga hermonthica (Delile) Benth (42,3\%) sont les plus rencontrées dans la zone d'étude. Les thérophytes représentent le type biologique le plus dominant.

Le sarclo-binage manuel (avec la daba) et mécanique (avec la houe-sine) est pratiqué sur la majorité des parcelles (73\%). Cependant $80 \%$ des parcelles à Dioulacolon ne sont pas désherbées Tableau 8.
Les principales maladies rencontrées dans les parcelles de mil sanio sont le mildiou (Sclerospora graminicola) et le charbon (Tolysposporium pennicillariae). Les insectes sont presque rencontrés dans toutes les parcelles suivies. Toutefois, les cantharides comme Cantharis fusca sont les plus évoqués par les producteurs à cause des dégâts qu'ils occasionnent sur les épis.

\section{Analyse des rendements}

Les parcelles de mil sanio sont caractérisées par une grande variabilité des rendements d'une localité à une autre Tableau 9. Les rendements les plus élevés ont été observés dans les localités de Guiro Yero Bocar (630 $\pm 167 \mathrm{~kg}$ ha-1), de Médina El Hadji (626 $\pm 133 \mathrm{~kg}$ ha-1) et de Bignarabe (540 $\pm 63,7 \mathrm{~kg}$ ha-1). Par contre, les plus faibles rendements ont été notés dans la commune de Ndorna (369 $\pm 103 \mathrm{~kg}$ ha- 1$)$. Sur les faibles superficies $(<1 \mathrm{ha})$, le rendement tournait autour de $296 \mathrm{~kg}$ ha-1 et est majoritairement localisé dans les communes de Bignarabe et Ndorna.

Tableau 2: Répartition (\%) des parcelles de mil sanio en fonction du type de champ et de la superficie emblavée.

\begin{tabular}{|c|c|c|c|c|c|c|}
\hline \multirow[b]{2}{*}{ Commune } & \multicolumn{6}{|c|}{ Pourcentage des parcelles } \\
\hline & $\begin{array}{l}\text { Champ de } \\
\text { case }\end{array}$ & $\begin{array}{l}\text { Champ de } \\
\text { brousse }\end{array}$ & $<1$ ha & [1-2 ha [ & [2-3 ha [ & $\geq 3$ ha \\
\hline Ndorna $(\mathrm{n}=5)$ & 40 & 60 & 75 & 25 & 0 & 0 \\
\hline Bignarabe $(n=5)$ & 20 & 80 & 20 & 40 & 40 & 0 \\
\hline Dioulacolon $(\mathrm{n}=5)$ & 20 & 80 & 60 & 20 & 20 & 0 \\
\hline $\begin{array}{l}\text { Guiro Yere Bocar } \\
(\mathrm{n}=5)\end{array}$ & 83 & 17 & 0 & 100 & 0 & 0 \\
\hline $\begin{array}{l}\text { Medina } \\
(\mathrm{n}=6)\end{array}$ & 0 & 100 & 33 & 50 & 0 & \\
\hline Moyenne & 33 & 67 & 38 & 47 & 12 & 3 \\
\hline
\end{tabular}

Tableau 3 : Répartition (\%) des parcelles de mil sanio en fonction du précédent cultural.

\begin{tabular}{lllll}
\hline Commune & \multicolumn{3}{l}{ Pourcentage des parcelles } & \\
\cline { 2 - 5 } & Arachide & Maïs & Mil sanio & Jachère \\
\hline Ndorna $(\mathrm{n}=5)$ & 60 & 20 & 0 & 20 \\
Bignarabe $(\mathrm{n}=5)$ & 20 & 0 & 60 & 20 \\
Dioulacolon $(\mathrm{n}=5)$ & 100 & 0 & 0 & 0 \\
Guiro Yero Bocar $(\mathrm{n}=5)$ & 60 & 40 & 0 & 0 \\
Medina El Hadj $(\mathrm{n}=6)$ & 16 & 50 & 17 & 17 \\
\hline Moyenne & 51 & 22 & 15 & 11 \\
\hline
\end{tabular}


Tableau 4: Répartition des parcelles de mil sanio en fonction du mode et de la période de semis.

\begin{tabular}{llllllll}
\hline \multirow{2}{*}{ Commune } & \multicolumn{6}{c}{ Pourcentage des parcelles } \\
\cline { 2 - 7 } & $\begin{array}{l}\text { Semis } \\
\text { plat }\end{array}$ & $\begin{array}{l}\text { à } \\
\text { Semis } \\
\text { billons }\end{array}$ & sur & $\begin{array}{l}{\left[\mathbf{1}^{\text {er }}-\mathbf{1 5}\right.} \\
\text { juin }[\end{array}$ & $\begin{array}{l}{[\mathbf{1 5 - 3 0}} \\
\text { juin }[\end{array}$ & $\begin{array}{l}{\left[\mathbf{1}^{\text {er }}-\mathbf{1 5}\right.} \\
\text { juillet }[\end{array}$ & {$[\mathbf{1 5 - 3 1}$ juillet [} \\
\hline Ndorna (n=5) & 100 & 0 & 0 & 0 & 0 & 100 \\
Bignarabe (n=5) & 100 & 0 & 40 & 20 & 20 & 20 \\
$\begin{array}{l}\text { Dioulacolon (n=5) } \\
\text { Guiro Yero Bocar } \\
\text { (n=5) }\end{array}$ & 100 & 0 & 0 & 100 & 0 & 0 \\
Medina El Hadj (n=6) & 100 & 0 & 0 & 60 & 40 & 0 \\
\hline Moyenne & 67 & 33 & 16 & 34 & 0 & 50 \\
\hline
\end{tabular}

Tableau 5 : Répartition des producteurs en fonction de la fumure.

\begin{tabular}{lll}
\hline \multirow{2}{*}{ Intrants } & \multicolumn{2}{l}{ Pourcentage des producteurs } \\
\cline { 2 - 3 } & Oui & Non \\
\hline NPK $(15-15-15)$ & 23 & 77 \\
Urée $(46-0-0)$ & 19 & 81 \\
Bouses de vache & 38 & 62 \\
\hline
\end{tabular}

Tableau 6: Répartition des parcelles de mil sanio en fonction du recouvrement de l'engrais.

\begin{tabular}{|c|c|c|}
\hline \multirow[b]{2}{*}{ Commune } & \multicolumn{2}{|c|}{ Pourcentage des parcelles } \\
\hline & $\begin{array}{l}\text { Recouvrement } \\
\text { immédiat }\end{array}$ & $\begin{array}{l}\text { Pas de } \\
\text { recouvrement }\end{array}$ \\
\hline Ndorna $(\mathrm{n}=5)$ & 0 & 100 \\
\hline Bignarabe $(n=5)$ & 100 & 0 \\
\hline Dioulacolon $(\mathrm{n}=5)$ & 100 & 0 \\
\hline Guiro Yero Bocar $(n=5)$ & 67 & 33 \\
\hline Medina El Hadj $(n=6)$ & 100 & 0 \\
\hline Moyenne & 73 & 27 \\
\hline
\end{tabular}

Tableau 7: Diversité spécifique, fréquence d'occurrence, type biologique des adventices rencontrées dans les parcelles de mil sanio.

\begin{tabular}{llll}
\hline Famille & Espèce (n=8) & $\begin{array}{l}\text { Fréquence } \\
\text { d'occurrence (\%) }\end{array}$ & $\begin{array}{l}\text { Type } \\
\text { Biologique. }\end{array}$ \\
\hline Asteraceae (D) & Acanthospermum hispidum DC & 3,8 & $\mathrm{~T}$ \\
Commelinaceae (M) & Commelina benghalensis L. & 3,8 & $\mathrm{~T}$ \\
Lamiaceae (D) & Hyptis suaveonlens (L.) Poit. & 3,8 & $\mathrm{~T}$ \\
Orobanchaceae (D) & Striga hermonthica (Delile) Benth. & 42,3 & $\mathrm{Par}$ \\
& Digitaria horizontalis Willd. & 88,5 & $\mathrm{~T}$ \\
Poaceae (M) & Dactyloctenium aegyptium Bauv. & 19,2 & $\mathrm{~T}$ \\
& Pennisetum pedicellatum Trin. & 57,7 & $\mathrm{~T}$ \\
Rubiaceae (D) & Spermacoce stachydea (DC.) Hutch. Et & 15,4 & $\mathrm{~T}$ \\
& Dalz. & & \\
\hline
\end{tabular}

M= monocotylédone; $\mathrm{D}=$ dicotylédone; $\mathrm{T}=$ térophytes ; Par= Parasite 
Tableau 8: Répartition des parcelles de mil sanio en fonction des travaux d'entretien.

\begin{tabular}{llll}
\hline \multirow{2}{*}{ Commune } & \multicolumn{2}{l}{ Pourcentage des parcelles } \\
\cline { 2 - 4 } & $\begin{array}{l}\text { Désherbage } \\
\text { mécanique }\end{array}$ & Désherbage manuel & $\begin{array}{l}\text { Pas de } \\
\text { désherbage }\end{array}$ \\
\hline Ndorna $(\mathrm{n}=5)$ & 100 & 0 & 0 \\
Bignarabe $(\mathrm{n}=5)$ & 0 & 60 & 40 \\
Dioulacolon (n=5) & 0 & 20 & 80 \\
Guiro Yero Bocar $(\mathrm{n}=5)$ & 60 & 40 & 0 \\
Medina El Hadj $(\mathrm{n}=6)$ & 0 & 83 & 17 \\
\hline Moyenne & 32 & 41 & 27 \\
\hline
\end{tabular}

Tableau 9: Rendements grains $(\mathrm{kg} / \mathrm{ha})$ des parcelles en fonction de la superficie cultivée.

\begin{tabular}{|c|c|c|c|c|}
\hline \multirow[t]{2}{*}{ Commune } & \multirow[t]{2}{*}{ Rendement en grains $\left(\mathrm{kg} \mathrm{ha}^{-1}\right)$} & \multicolumn{3}{|c|}{$\begin{array}{l}\text { Rendement en grain }\left(\mathrm{kg} \mathrm{ha}^{-1}\right) \text { en } \\
\text { fonction de la superficie cultivée }\end{array}$} \\
\hline & & $<1$ ha & [1-2 ha [ & $>2$ ha \\
\hline Ndorna $(n=5)$ & $369 \pm 103$ & 232 & 90 & 0 \\
\hline Bignarabe $(n=5)$ & $540 \pm 63$ & 750 & 328 & 288 \\
\hline Dioulacolon $(\mathrm{n}=5)$ & $516 \pm 244$ & 246 & 20 & 717 \\
\hline Guiro Yero Bocar $(n=5)$ & $630 \pm 166$ & 0 & 399 & 0 \\
\hline Medina El hadj (n=6) & $626 \pm 133$ & 251 & 414 & 333 \\
\hline Moyenne & $536 \pm 142$ & 296 & 251 & 268 \\
\hline
\end{tabular}

\section{DISCUSSION}

L'étude a montré que le mil sanio est plus cultivé dans les champs de brousse que ceux de case. Cette situation se justifie par le fait que les champs de case sont principalement consacrés aux cultures bénéficiant d'une gestion de la fertilité des sols par l'utilisation de la fumure animale et par l'apport d'ordures ménagères (Muller et al., 2015). Les superficies emblavées sont très variables allant de moins d'un à trois hectares avec $77 \%$ des parcelles faisant moins de deux hectares. Les travaux de Fofana et Mbaye (1990) ont montré que le mil sanio n'occupe que $15 \%$ des superficies totales emblavées en mil au Sénégal. La place marginale, l'absence d'accompagnement par la recherche et plus récemment les effets néfastes du changement climatique pourraient être des éléments explicatifs des faibles superficies consacrées à cette culture dans la zone malgré les potentialités existantes (Sall et al., 2010). Dans la zone du bassin arachidier, les producteurs de mil souna ont en moyenne 4, 9 ha contre 4,2 ha pour les autres cultures (Muller et al., 2015).

Dans toute la zone d'étude, l'arachide est le principal précèdent cultural du mil sanio. Cela est lié à la fonction que cette légumineuse joue dans la restauration de la fertilité des sols. En effet, l'arachide fixe l'azote atmosphérique à travers la symbiose racinaire avec les rhizobiums et permet ainsi une gestion de la fertilité du sol. Il a été montré l'importance agronomique des légumineuses comme précédent cultural des 
céréales (Ngoran et Kanga, 2000 ; Cattan et al., 2001). Les travaux de Bationo et Ntare (2000) ont démontré que la rotation légumineuse-mil avec un apport de $30 \mathrm{~kg} \mathrm{ha}^{-1}$ d'urée permet l'obtention de bons rendements en grain chez le mil.

Le suivi des adventices a montré une prédominance des dicotylédones et des thérophytes dans la zone d'étude. En effet, plusieurs études floristico-écologiques ont montré la dominance des thérophytes (espèces annuelles) dans les systèmes de culture à base de céréales au Sénégal (Noba, 2002 ; Mbaye, 2012 ; Bassène, 2014) et au Maroc (Zidane et al., 2010). Le Striga hermontica est rapporté comme étant l'adventice la plus répandue dans les champs de brousse. Le Striga est connu comme un indicateur de baisse de la fertilisé des sols et sa présence est étroitement liée au statut nutritionnel du sol (Lopez-Raez et al., 2008). Les travaux de Wade et Kamara (2007) au Sénégal ont montré que le Striga a causé sur les mil Souna des pertes de rendement variant entre 30 et $52 \%$.

La faible utilisation des fertilisants chimiques et organiques peut s'expliquer en grande partie par la cherté et l'inaccessibilité des engrais chimiques et d'autre part par l'insuffisance du fumier. L'effet positif de l'application conjointe de la fumure minérale et de la fumure organique a été noté sur le maïs (Nyami et al., 2014), le mil (Zeinabou et al., 2014), le sorgho (Somda et al., 2017).

Dans la zone d'étude, le rendement moyen en grains était de $536 \mathrm{~kg} \mathrm{ha}^{-1}$, ce qui est inférieur à la moyenne nationale qui est de $811 \mathrm{~kg} \mathrm{ha}^{-1}$ (ANSD, 2014). Les rendements grain les plus élevés ont été observés dans les communes de Guiré Yero Bocar, Bignarabe, Dioulacolon et Médina El Hadji tandis que les plus faibles productions ont été notés à Ndorna. Généralement les facteurs qui expliquent ces mauvais rendements sont d'ordre abiotique (déficit pluviométrique, baisse de la fertilité des sols) biotique (forte pression des adventices et des maladies, matériel végétal local peu productif), technique (pratiques culturales traditionnelles) et le sous-équipement en matériel agricole. Dans la zone du bassin arachidier, les rendements du mil Souna en milieu réel ont oscillé en moyenne entre 500 et $700 \mathrm{~kg} / \mathrm{ha}$ durant les deux dernières décennies du fait de nombreuses contraintes (Kouakou et al., 2013).

\section{Conclusion}

Cette étude qui fait un diagnostic des pratiques culturales du mil sanio en parcelles paysannes en zone soudanienne humide au Sénégal a permis de mieux connaitre les facteurs limitant le rendement de cette culture. En effet, le mil est largement cultivé dans les champs de brousse sur de faibles superficies. La période de semis est calée dans la deuxième quinzaine des mois de juin et de juillet. Les techniques de désherbage sont manuelle et mécanique, la fertilisation et/ou l'amendement sont des pratiques peu répandues dans la zone. Les faibles rendements obtenus $(<700 \mathrm{~kg} / \mathrm{ha})$ sont entre autres dus à l'utilisation d'un matériel végétal peu productif et à l'absence d'un référentiel technique adapté aux conditions du milieu.

\section{CONFLIT D'INTERETS}

Les auteurs déclarent qu'il n'y a aucun conflit d'intérêt.

\section{CONTRIBUTIONS DES AUTEURS}

BB participé à l'élaboration du protocole de recherche, à la collecte des données, à l'analyse statistique et à la rédaction de l'article. MG et DNG ont contribué à la correction de l'article. SLK, BD et GK ont contribué à la collecte des données sur le terrain.

\section{REMERCIEMENTS}

Nous adressons nos sincères remerciements au Programme de Productivité Agricole en Afrique de l'Ouest (PPAAO), aux collègues des Centres de Recherches Agricoles de Djbélor et Zootechniques de Kolda et de l'Université Cheikh Anta Diop de Dakar.

\section{REFERENCES}

ANSD. 2014. Bulletin mensuel des statistiques économiques, Décembre 2014. ISSN 0850-1467. 
ANSD. 2015. Situation économique et sociale de la région de Kolda en 2013. Service Régional de la Statistique et de la démographie, p 99.

Bagayoko M, Buerker A, Lung G, Bationo A, Römheld V. 2000. Cereal /legume rotation effects on cereal growth in Soudano-Sahelian West Africa: soil mineral nitrogen, mycorrhizae and nematodes. Plant and Soils 218. 103116, 2000. (C) 2000 Kluwer Academic Publishers. Printed in the Netherlands.

Bassène C. 2014. La flore adventice dans les cultures de maïs (Zeas mays L.) dans le sud du Bassin arachidier : structure, nuisibilité et mise au point d'un itinéraire de désherbage. Thèse de Doctorat, Faculté des Sciences et Techniques, Université Cheikh Anta Diop, Dakar (Sénégal), p 149 + Annexes.

Bationo A, Ntare BR. 2000. Rotation and nitrogen fertilizer effects on pearl millet, cowpea and groundnut yield and soil chemical properties in a sandy soil in the semi-arid tropics. Journal of Agricultural Science, 134: 277-284.

Berhaut J. 1967. Flore du Sénégal (2ème Edition). Clairafrique : Dakar (Sénégal) ; p 257.

Cattan Ph, Letourmy Ph, Zagre B, Minougou A, Compaoré E. 2001. Rendement de l'arachide et du sorgho en rotation sous différents itinéraires techniques au Burkina Faso. Cahiers agricultures, 10 : 59-72 p.

Fall AA, Lo M. 2009. Etude de référence du Programme sur la productivité agricole au Sénégal dans le cadre du WAAPP. Cas des céréales : mil, sorgho, maïs et fonio. CORAF, PSAOP II, $\mathrm{p} 38$.

Fofana A, Mbaye DF. 1990. Production du mil au Sénégal: contraintes et perspectives de de recherches. In Proceedings of the Regional Pearl Millet Workshop. ICRISAT Sahelian Center, Niamey-Niger, p 134-141.

Fofana A, Tall H, Guèye M, Badiane D, Guèye M, Sow DS, Sall M. 2009. Amélioration de la productivité du mil au Sénégal Oriental et en Casamance. Rapport annuel 2008, p 35.

ISRA, ITA, CIRAD. 2005. Bilan de la recherche agricole et agroalimentaire au Sénégal. Institut sénégalais de recherches agricoles, p 524.

Kouakou PK, Muller B. Guissé A. Yao RN, Fofana A, Cissé N. 2013. Étude et prise en compte en modélisation de l'effet de la latitude sur la réponse à la photopériode chez divers génotypes de mil (Pennisetum glaucum (L.) R. Br.) du Sénégal. J. Appl. Biosci., 67: 5289-5301. DOI :

www.m.elewa.org/JABS/2013/67/Abstra ct12-kouakou.html.

Lebrun J. 1966. Les formes biologiques dans les végétations tropicales. Bull. Sco. Bot. France: 164- 175.

Lebrun JP, Stork A. 1997. Enumération des plantes à fleurs d'Afrique Tropicale. Vol I, Vol II, Vol III, Vol IV. Edition des conservatoires et jardin botaniques de la ville de Genève.

Lopez-Raez, JA, Charnikhova T, GomezRoldan V, Gómez-Roldán V, Matusova R, Kohlen W, De Vos R, Verstappen F, Puech-Pages V, Bécard G, Mulder P, Bouwmeester H. 2008. Tomato strigolactones are derived from carotenoids and their biosynthesis is promoted by phosphate starvation. New Phytol., 178: 863-874. DOI: https://doi.org/10.1111/j.14698137.2008.02406.x

Mbaye MS. 2013. Association mil [Pennisetum glaucum (L.) R. Br.] et niébé [Vigna unguiculata(L.) Walp.]: Arrangement spatio-temporel des cultures, structure, dynamique et concurrence de la flore adventice et proposition d'un itinéraire technique. Thèse d'Etat ès Sciences Naturelle, Faculté des Sciences et Techniques, Université Cheikh Anta Diop, Dakar (Sénégal), p 227 +Annexes.

Merlier H, Montegut J. 1982. Adventices tropicales. Flore aux stades plantule et adulte de 123 espèces africaines ou 
pantropicales. ORSTOM-GERDATENSH Paris (France), p 490.

Muller B, Lalou R, Kouakou P, Soumaré MA, Bourgoin J, Dorégo S, Sine B. 2015. Le retour du mil sanio dans le Sine: Une adaptation raisonnée à l'évolution climatique, p 377-401. In Les sociétés rurales face aux changements environnementaux en Afrique de l'Ouest, Conférence ESCAPE, 29-30 janvier 2015, Université Pierre et Marie Curie, Paris

Ngoran A, Kanga ANg. 2000. Influence d'un précédent de légumineuse herbacée et d'une jachère courte de deux ans sur la productivité du maïs ai nord de la Côte d'Ivoire. In $\mathrm{La}$ jachère en Afrique tropicale : rôles, aménagement et alternatives, 616-621. Floret $\mathrm{Ch}$. et Pontanier R.(Eds). Actes du séminaire international, 13-16 avril 1999, Dakar (Sénégal). John Libbey Eurotext, Paris (France).

Noba K. 2002. La flore adventice dans le sud du Bassin arachidier (Sénégal) : structure, dynamique et impact sur la production du mil et de l'arachide. Thèse de doctorat d'état en sciences naturelles. Faculté des Sciences et Techniques, Université Cheikh Anta Diop, Dakar (Sénégal), p 128.

Nyami BL, Sudi CK, Lejoly J. 2014. Effet du biochar et des feuilles de Tithonia diversifolia combiné à l'engrais minéral sur la culture du maïs (Zea mays L.) et les propriétés d'un sol ferralitique à Kinshasa (RDC). BASE-volume 20 (2016) Numéro 1

Raunkier C. 1934. The life from of plants and statistical plants geography. Clarendron, Press, Oxford, p 623.

ROCAFREMI. 2002. Sélection et Mise à Disposition des Paysans de Variétés et de Semences Appropriées. Des Résultats du Projet P1 : 1991-1996.
Sagna P, Yade M, Sambou PC. 2012. Migrations de l'équateur météorologique, fréquences de la mousson et importance des précipitations au Sénégal en 2008 et 2009. Annales de la faculté des lettres et sciences humaines $\mathrm{N}^{\circ}$ 42/B -2012, Université Cheikh Anta Diop.

Sall M, Gafsi M, Bonnaissiux A. 2010. Les systèmes de production dans la région de Kolda : Dynamique des innovations à travers l'aviculture villageoise. Montpellier, France, p 13.

Somda BB, Ouattara AB, Serme I, Pouya MB, Lompo F, Taonda. SJP, Sedogo PM. 2017. Détermination des doses optimales de fumures organo-minérales en microdose dans la zone soudanosahélienne du Burkina Faso. Int. J. Biol. Chem. Sci., 11(2): 670-683. http://ajol.info/index.php/ijbcs

Trochain JL. 1966. Types biologiques chez les végétaux intertropicaux (Angiospermes). Bull. Soc. Bot. de France: 188-196. DOI: 10.1080/00378941.1966.10838486.

Wade I, Kamara O. 2007. Formation de Formateurs et de producteurs sur la lutte integrée durable contre Striga au Sénégal, In Progress on Farmers Training on Parasitic Weed Mangement. Ricardo Labrada, FAO, Rome, p. 129138.

Zeinabou H, Mahamane S, Bismarck NH, Bado BV, Lompo F, Bationo A. 2014. Effet de la combinaison des fumures organo-minérales et de la rotation niébémil sur la nutrition azotée et les rendements du mil au sahel. International Journal of Biological and Chemical Sciences, 8(4): 1620-1632. http://ajol.info/index.php/ijbcs.

Zidane L, Salhi S, Fadli M, El Antri M, Taleb A, Douira A. 2010. Etude des groupements d'adventices dans le Maroc occidental. Biotechnol. Agron. Soc. Environ., 14(1): 153-166. 